\title{
Security of Random Feistel Schemes with 5 or More Rounds
}

\author{
Jacques Patarin \\ Université de Versailles \\ 45 avenue des Etats-Unis \\ 78035 Versailles Cedex, France
}

\begin{abstract}
We study cryptographic attacks on random Feistel schemes. We denote by $m$ the number of plaintext/ciphertext pairs, and by $k$ the number of rounds. In their famous paper [3], M. Luby and C. Rackoff have completely solved the cases $m \ll 2^{n / 2}$ : the schemes are secure against all adaptive chosen plaintext attacks (CPA-2) when $k \geq 3$ and against all adaptive chosen plaintext and chosen ciphertext attacks (CPCA-2) when $k \geq 4$ (for this second result a proof is given in [9]).

In this paper we study the cases $m \ll 2^{n}$. We will use the "coefficients $H$ technique" of proof to analyze known plaintext attacks (KPA), adaptive or non-adaptive chosen plaitext attacks (CPA-1 and CPA-2) and adaptive or non-adaptive chosen plaitext and chosen ciphertext attacks (CPCA-1 and CPCA-2). In the first part of this paper, we will show that when $m \ll 2^{n}$ the schemes are secure against all KPA when $k \geq 4$, against all CPA-2 when $k \geq 5$ and against all CPCA-2 attacks when $k \geq 6$. This solves an open problem of [1], [14], and it improves the result of [14] (where more rounds were needed and $m \ll 2^{n(1-\varepsilon)}$ was obtained instead of $\left.m \ll 2^{n}\right)$. The number 5 of rounds is minimal since CPA-2 attacks on 4 rounds are known when $m \geq O\left(2^{n / 2}\right)$ (see [1], [10]). Furthermore, in all these cases we have always obtained an explicit majoration for the distinguishing probability. In the second part of this paper, we present some improved generic attacks. For $k=5$ rounds, we present a KPA with $m \simeq 2^{3 n / 2}$ and a non-adaptive chosen plaintext attack (CPA1) with $m \simeq 2^{n}$. For $k \geq 7$ rounds we also show some improved attacks against random Feistel generators (with more than one permutation to analyze and $\geq 2^{2 n}$ computations).
\end{abstract}

\section{Introduction}

A "Luby - Rackoff construction with $k$ rounds", which is also known as a "random Feistel cipher" is a Feistel cipher in which the round functions $f_{1}, \ldots, f_{k}$ are independently chosen as truly random functions (see section 2 for precise definitions).

Since the famous original paper [3] of M. Luby and C. Rackoff, these constructions have inspired a considerable amount of research. In [8] and [14] a summary of existing works on this topic is given.

We will denote by $k$ the number of rounds and by $n$ the integer such that the Feistel cipher is a permutation of $2 n$ bits $\rightarrow 2 n$ bits. In [3] it was proved 
that when $k \geq 3$ these Feistel ciphers are secure against all adaptative chosen plaintext attacks (CPA-2) when the number of queries (i.e. plaintext/ciphertext pairs obtained) is $m \ll 2^{n / 2}$. Moreover when $k \geq 4$ they are secure against all adaptative chosen plaintext and chosen ciphertext attacks (CPCA-2) when the number of queries is $m \ll 2^{n / 2}$ (a proof of this second result is given in [9]).

These results are valid if the adversary has unbounded computing power as long as he does only $m$ queries.

These results can be applied in two different ways: directly using $k$ truly random functions $f_{1}, \ldots, f_{k}$ (that requires significant storage), or in a hybrid setting, in which instead of using $k$ truly random functions $f_{1}, \ldots, f_{k}$, we use $k$ pseudo-random functions. These two ways are both interesting for cryptography. The first way gives "locally random permutations" where we have proofs of security without any unproven hypothesis (but we need a lot of storage), and the second way gives constructions for block encryption schemes where the security can be relied on a pseudo-random number generator, or on any one-way function.

In this paper, we will study security when $m \ll 2^{n}$, instead of $m \ll 2^{n / 2}$ for the original paper of M. Luby and C. Rackoff. For this we must have $k \geq 5$, since for $k \leq 4$ some CPA-2 attacks when $m \geq O\left(2^{n / 2}\right)$ exist (see [1], [10]). Moreover the bound $m \ll 2^{n}$ is the larger bound that we can get, since an adversary with unlimited computing power can always distinguish a $k$-round random Feistel scheme from a random permutation with $O\left(k \cdot 2^{n}\right)$ queries and $O\left(2^{k n 2^{n}}\right)$ computations by simply guessing all the round functions (it is also possible to do less computing with the same number of queries by using collisions, see [13]).

The bound $m \ll 2^{n / 2}$ is called the 'birthday bound', i.e. it is about the square root of the optimal bound against an adversary with unbounded computing power. In [1] W. Aiello and R. Venkatesan have found a construction of locally random functions ('Benes') where the optimal bound $\left(m \ll 2^{n}\right.$ ) is obtained instead of the birthday bound. However here the functions are not permutations. Similarly, in [4], U. Maurer has found some other construction of locally random functions (not permutations) where he can get as close as wanted to the optimal bound (i.e. $m \ll 2^{n(1-\epsilon)}$ and for all $\epsilon>0$ he has a construction). In [8] the security of unbalanced Feistel schemes is studied and a security proof in $2^{n(1-\epsilon)}$ is obtained, instead of $2^{n / 2}$, but for much larger round functions (from $2 n$ bits to $\epsilon$ bits, instead of $n$ bits to $n$ bits). This bound is basically again the birthday bound for these functions.

In this paper we will show that 5-round random Feistel schemes resist all CPA-2 attacks when $m \ll 2^{n}$ and that 6 -round random Feistel schemes resist all CPCA-2 attacks when $m \ll 2^{n}$. Here we are very near the optimal bound, and we have permutations. This solves an open problem of [1], [10]. It also significantly improves the results of [6] in which the $2^{n}$ security is only obtained when the number of rounds tends to infinity, and the result of [14] where $2^{n(1-\epsilon)}$ security was proved for CPA-2 after 7 rounds (instead of 5 here) and for CPCA-2 after 10 rounds (instead of 6 here). Moreover we will obtain in this paper some explicit and simple majorations for the distinguishing probabilities. We will also present some improved generic attacks. All these results are summarized in appendix A. 


\section{Notations}

\section{General notations}

- $I_{n}=\{0,1\}^{n}$ denotes the set of the $2^{n}$ binary strings of length $n .\left|I_{n}\right|=2^{n}$.

- The set of all functions from $I_{n}$ to $I_{n}$ is $F_{n}$. Thus $\left|F_{n}\right|=2^{n \cdot 2^{n}}$.

- For any $f, g \in F_{n}, f \circ g$ denotes the usual composition of functions.

- For any $a, b \in I_{n},[a, b]$ will be the string of length $2 n$ of $I_{2 n}$ which is the concatenation of $a$ and $b$.

- For $a, b \in I_{n}, a \oplus b$ stands for bit by bit exclusive or of $a$ and $b$.

- Let $f_{1}$ be a function of $F_{n}$. Let $L, R, S$ and $T$ be four n-bit strings in $I_{n}$. Then by definition

$$
\Psi\left(f_{1}\right)[L, R]=[S, T] \stackrel{\text { def }}{\Leftrightarrow}\left\{\begin{array}{l}
S=R \\
T=L \oplus f_{1}(R)
\end{array}\right.
$$

- Let $f_{1}, f_{2}, \ldots, f_{k}$ be $k$ functions of $F_{n}$. Then by definition:

$$
\Psi^{k}\left(f_{1}, \ldots, f_{k}\right)=\Psi\left(f_{k}\right) \circ \cdots \circ \Psi\left(f_{2}\right) \circ \Psi\left(f_{1}\right) .
$$

The permutation $\Psi^{k}\left(f_{1}, \ldots, f_{k}\right)$ is called a 'Feistel scheme with $k$ rounds' or shortly $\Psi^{k}$. When $f_{1}, \ldots, f_{k}$ are randomly and independently chosen in $F_{n}$, then $\Psi^{k}\left(f_{1}, \ldots, f_{k}\right)$ is called a 'random Feistel scheme with $k$ rounds' or a 'LubyRackoff construction with $k$ rounds'.

We will first study 4 rounds (with some limitations on the inputs/outputs), then prove our cryptographic results by adding one or two rounds.

Notations for 4 rounds

- We will denote by $\left[L_{i}, R_{i}\right], 1 \leq i \leq m$, the $m$ cleartexts. These cleartexts can be assumed to be pairwise distinct, i.e. $i \neq j \Rightarrow L_{i} \neq L_{j}$ or $R_{i} \neq R_{j}$.

- We call "index" any integer between 1 and $m$.

- $\left[R_{i}, X_{i}\right]$ is the output after one round, i.e.

$$
\forall i, 1 \leq i \leq m, X_{i}=L_{i} \oplus f_{1}\left(R_{i}\right) .
$$

- $\left[X_{i}, Y_{i}\right]$ is the output after two rounds, i.e.

$$
\forall i, 1 \leq i \leq m, Y_{i}=R_{i} \oplus f_{2}\left(X_{i}\right)=R_{i} \oplus f_{2}\left(L_{i} \oplus f_{1}\left(R_{i}\right)\right) .
$$

- $\left[Y_{i}, S_{i}\right]$ is the output after three rounds, i.e.

$$
\forall i, 1 \leq i \leq m, S_{i}=X_{i} \oplus f_{3}\left(Y_{i}\right)=L_{i} \oplus f_{1}\left(R_{i}\right) \oplus f_{3}\left(Y_{i}\right) .
$$

- $\left[S_{i}, T_{i}\right]$ is the output after 4 rounds, i.e.

$$
\forall i, 1 \leq i \leq m, T_{i}=Y_{i} \oplus f_{4}\left(S_{i}\right) .
$$

Notations for 5 rounds. We keep the same notations for $L_{i}, R_{i}, X_{i}, Y_{i}$. Now $Z_{i}=X_{i} \oplus f_{3}\left(Y_{i}\right)$, and $\left[S_{i}, T_{i}\right]$ is still the output: $S_{i}=Y_{i} \oplus f_{4}\left(Z_{i}\right)$ and $T_{i}=$ $Z_{i} \oplus f_{5}\left(S_{i}\right)$. 


\section{Part I: Security Results}

\section{The General Proof Strategy}

We will first study the properties of 4-round schemes. Our result on 4-round schemes for proving KPA security will be:

Theorem 3.1 (4 rounds) For random values $\left[L_{i}, R_{i}\right],\left[S_{i}, T_{i}\right], 1 \leq i \leq m$, such that the $\left[L_{i}, R_{i}\right], 1 \leq i \leq m$, are pairwise distinct, with probability $\geq 1-\beta$ we have:

1. the number $H$ of $\left(f_{1}, f_{2}, f_{3}, f_{4}\right) \in F_{n}^{4}$ such that $\forall i, 1 \leq i \leq m$,

$$
\Psi^{4}\left(f_{1}, f_{2}, f_{3}, f_{4}\right)\left[L_{i}, R_{i}\right]=\left[S_{i}, T_{i}\right]
$$

satisfies:

$$
H \geq \frac{\left|F_{n}\right|^{4}}{2^{2 n m}}(1-\alpha) .
$$

2. $\alpha$ and $\beta$ can be chosen $\ll 1$ when $m \ll 2^{n}$.

For 5 rounds, we will have:

Theorem 3.2 ( 5 rounds) There are some values $\alpha>0$ and $\beta>0$ and there is a subset $E \subset I_{2 n}^{m}$ such that:

1. for all pairwise distinct $\left[L_{i}, R_{i}\right], 1 \leq i \leq m$, and for all sequences $\left[S_{i}, T_{i}\right]$, $1 \leq i \leq m$, of $E$ the number $H$ of $\left(f_{1}, f_{2}, f_{3}, f_{4}, f_{5}\right) \in F_{n}^{5}$ such that $\forall i$, $1 \leq i \leq m$,

$$
\Psi^{5}\left(f_{1}, f_{2}, f_{3}, f_{4}, f_{5}\right)\left[L_{i}, R_{i}\right]=\left[S_{i}, T_{i}\right]
$$

satisfies:

$$
H \geq \frac{\left|F_{n}\right|^{5}}{2^{2 n m}}(1-\alpha)
$$

2. $|E| \geq(1-\beta) \cdot 2^{2 n m}$, and $\alpha$ and $\beta$ can be chosen $\ll 1$ when $m \ll 2^{n(1-\varepsilon)}$, $\forall \varepsilon>0$.

Remark

1. Here the set $E$ does not depend on the $\left[L_{i}, R_{i}\right]$, and it will give security against CPA-2. If $E$ depends on the $\left[L_{i}, R_{i}\right]$, we will obtain security against CPA-1 only.

2. Instead of fixing a set $E$, as in theorem 3.2 , we can formulate a similar theorem in term of expectancy of the deviation of $H$ from the average value (see[15]: there is a formulation for CPA-1 and another for CPA-2). From these formulas we will get security when $m \ll 2^{n}$.

For 6 rounds, we will have: 
Theorem 3.3 (6 rounds) There are some values $\alpha>0$ and $\beta>0$ and there is a subset $E \subset I_{n}^{4 m}$ such that:

1. for all $\left[L_{i}, R_{i}, S_{i}, T_{i}\right], 1 \leq i \leq m$, of $E$, the number $H$ of $\left(f_{1}, f_{2}, f_{3}, f_{4}, f_{5}\right.$, $\left.f_{6}\right) \in F_{n}^{6}$ such that $\forall i, 1 \leq i \leq m$,

$$
\Psi^{6}\left(f_{1}, f_{2}, f_{3}, f_{4}, f_{5}, f_{6}\right)\left[L_{i}, R_{i}\right]=\left[S_{i}, T_{i}\right]
$$

satisfies:

$$
H \geq \frac{\left|F_{n}\right|^{6}}{2^{2 n m}}(1-\alpha) .
$$

2. For all super distinguishing circuit $\Phi$ with $m$ oracle gates, the probability that $\left[L_{i}, R_{i}, S_{i}, T_{i}\right](\Phi), 1 \leq i \leq m$, be in $E$ is $\geq 1-\beta$, when $\Phi$ acts on a random permutation $f$ of $I_{2 n} \rightarrow I_{2 n}$ (here $\left[L_{i}, R_{i}, S_{i}, T_{i}\right](\Phi), 1 \leq i \leq m$, denotes the successive $\left[S_{i}, T_{i}\right]=f\left[L_{i}, R_{i}\right]$ or $\left[L_{i}, R_{i}\right]=f^{-1}\left[S_{i}, T_{i}\right], 1 \leq i \leq m$, that will appear).

3. $\alpha$ and $\beta$ can be chosen $\ll 1$ when $m \ll 2^{n}$.

Now from these theorems and from the general "coefficients $H$ technique" theorems given in [11], [12], we will get immediately that when $m \ll 2^{n}, \Psi^{4}$ is secure against all KPA, $\Psi^{5}$ against all CPA-2 and $\Psi^{6}$ against all CPCA-2.

\section{Circles}

One of the terms of the the deviation of $\Psi^{k}$ from random permutations will be the probability to get "circles" in the variables, as we will explain below.

Definition. We will say that we have 'a circle in $R, X, Y$ ' if there are $k$ indices $i_{1}, \ldots, i_{k}$ with $k \geq 3$ and such that:

1. $i_{1}, i_{2}, \ldots, i_{k-1}$ are pairwise distinct and $i_{k}=i_{1}$.

2. $\forall \lambda, 1 \leq \lambda \leq k-2$ we have at least one of the three following conditions:

- $R_{i_{\lambda}}=R_{i_{\lambda+1}}$ and $\left(X_{i_{\lambda+1}}=X_{i_{\lambda+2}}\right.$ or $\left.Y_{i_{\lambda+1}}=Y_{i_{\lambda+2}}\right)$

or $\bullet X_{i_{\lambda}}=X_{i_{\lambda+1}}$ and $\left(R_{i_{\lambda+1}}=R_{i_{\lambda+2}}\right.$ or $\left.Y_{i_{\lambda+1}}=Y_{i_{\lambda+2}}\right)$

or $\bullet Y_{i_{\lambda}}=Y_{i_{\lambda+1}}$ and $\left(R_{i_{\lambda+1}}=R_{i_{\lambda+2}}\right.$ or $\left.X_{i_{\lambda+1}}=X_{i_{\lambda+2}}\right)$

Example. If $R_{1}=R_{2}$ and $X_{1}=X_{2}$, then we have a circle in $R, X, Y$. If $R_{1}=R_{2}$, $X_{2}=X_{3}, Y_{3}=Y_{1}$ then we have a circle in $R, X, Y$.

We will prove the following theorems.

Theorem 4.1 (For 4 rounds) When $\left[L_{i}, R_{i}\right], 1 \leq i \leq m$, are pairwise distinct and randomly chosen, the probability $p$ to obtain a circle in $R, X, Y$ with at least one equation in $Y$ when $f_{1}, f_{2}$ are randomly chosen in $F_{n}$ satisfies:

$$
p \leq \frac{3 m^{2}}{2 \cdot 2^{2 n}}+\frac{3 m^{3}}{2^{3 n}} \cdot \frac{1}{1-\frac{2 m}{2^{n}}} .
$$


Theorem 4.2 (For 5 rounds) For all pairwise distinct $\left[L_{i}, R_{i}\right], 1 \leq i \leq m$ and for all value $\lambda$, such that $\lambda>0$ and $2 m \sqrt{\lambda}<2^{n}$, we have: the probability $p$ to obtain a circle in $X, Y, Z$ with at least one equation $Z_{i}=Z_{j}$ when $f_{1}, f_{2}, f_{3}$ are randomly chosen in $F_{n}$ satisfies:

$$
p \leq \frac{1}{\lambda}+\frac{m(m-1)}{2 \cdot 2^{2 n}}+\frac{m(m-1)(m-2)}{2^{3 n}}+\frac{4 \lambda m^{4}}{2^{4 n}} \cdot \frac{1}{1-\frac{2 m \sqrt{\lambda}}{2^{n}}} .
$$

Corollary 4.1 From this theorem 4.2 we get immediately that if $m \ll 2^{n}$, then ( $\lambda$ can be chosen such that), $p$ is very small. So when $m \ll 2^{n}$, the probability to have a circle in $X, Y, Z$ with at least one equation $Z_{i}=Z_{j}$ is negligible.

Remark. In [15] we show that the condition 'with at least one equation $Z_{i}=Z_{j}$ ' is important: sometime we cannot avoid some circles in $X, Y$.

With 6 rounds, we can get a simpler formula:

Theorem 4.3 (For 6 rounds) For all $\left[L_{i}, R_{i}\right], 1 \leq i \leq m$ (such that $i \neq j \Rightarrow$ $L_{i} \neq L_{j}$ or $R_{i} \neq R_{j}$ ), the probability $p$ to obtain a circle in $X, Y, Z$ with at least one equation in $Z$ when $f_{1}, f_{2}, f_{3}, f_{4}$ are randomly chosen in $F_{n}$ satisfies:

$$
p \leq \frac{3 m^{2}}{2^{2 n}}+\frac{11 m^{3}}{2^{3 n}} \cdot \frac{1}{1-\frac{2 m}{2^{n}}} .
$$

Proof of theorem 4.1, 4.2, 4.3 are given in the extended version of this paper ([15]). A basic tool for these proofs is:

Theorem 4.4 $\forall \lambda>0$, for all pairwise distinct $\left[L_{i}, R_{i}\right], 1 \leq i \leq m$, when $f_{1}$ is randomly chosen in $F_{n}$ we have a probability $\geq 1-\frac{1}{\lambda}$ that the number $N$ of $(i, j), i<j / X_{i}=X_{j}$ satisfies:

$$
N \leq \frac{\lambda m(m-1)}{2 \cdot 2^{n}}
$$

Proof. This result comes immediately from this lemma:

Lemma 4.1 For all $\left[L_{i}, R_{i}\right], 1 \leq i \leq m$, (such that $i \neq j \Rightarrow L_{i} \neq L_{j}$ or $R_{i} \neq R_{j}$ ) the number of $\left(f_{1}, i, j\right)$ such that $X_{i}=X_{j}, i<j$, is $\leq\left|F_{n}\right| \cdot \frac{m(m-1)}{2 \cdot 2^{n}}$.

Proof of lemma 4.1. $X_{i}=X_{j}$ means $L_{i} \oplus f_{1}\left(R_{i}\right)=L_{j} \oplus f_{1}\left(R_{j}\right)$. This implies $R_{i} \neq R_{j}$ (because $L_{i}=L_{j}$ and $R_{i}=R_{j} \Rightarrow i=j$ ). Thus, when $(i, j)$ is fixed, the number of $f_{1}$ such that $X_{i}=X_{j}$ is exactly $\frac{\left|F_{n}\right|}{2^{n}}$ if $R_{i} \neq R_{j}$, and exactly 0 if $R_{i}=R_{j}$. Therefore, since we have at most $m(m-1) / 2$ values $(i, j), i<j / R_{i} \neq$ $R_{j}$, the total number of $\left(f_{1}, i, j\right)$ such that $X_{i}=X_{j}$ is $\leq\left|F_{n}\right| \frac{m(m-1)}{2 \cdot 2^{n}}$ as claimed. 


\section{Properties of $\boldsymbol{H}$ with 4 Rounds}

We give here the main ideas. See the extended version of this paper for more details ([15]). We will first prove that if the $\left[Y_{i}, S_{i}\right]$ are given, $1 \leq i \leq m$, (i.e. the output after 3 rounds), then the $S_{i}$ variables will look random as long as $m \ll 2^{n}$ (but the $Y_{i}$ variables will not look random in general). Then, with one more round and the same argument, we will obtain that the $\left[S_{i}, T_{i}\right]$ variables will look random as long as $m \ll 2^{n}$. We want to evaluate the number $H$ of $f_{1}, f_{2}, f_{3}$ such that: $\forall i, 1 \leq i \leq m, S_{i}=L_{i} \oplus f_{1}\left(R_{i}\right) \oplus f_{3}\left(Y_{i}\right)$ with $Y_{i}=R_{i} \oplus f_{2}\left(L_{i} \oplus f_{1}\left(R_{i}\right)\right)$

\section{Remarks}

1. If $Y_{i}=Y_{j}$ with $i \neq j$, then $S_{i} \neq S_{j}$. So the $S_{i}$ variables are not perfectly random in $I_{n}$ when the $Y_{i}$ are given. However, here we just say that the $\left[Y_{i}, S_{i}\right]$ must be pairwise distinct, since $\Psi^{k}$ is a permutation.

2. If $S_{i}$ is a constant $\left(\forall i, 1 \leq i \leq m, S_{i}=0\right.$ for example), then all the $Y_{i}$ variables must be pairwise distinct, and in (1) $f_{3}$ is then fixed on exactly $m$ points. However the probability for $f_{1}, f_{2}$ to be such that all the $Y_{i}$ are pairwise distinct is very small. So in this case $H \ll \frac{\left|F_{n}\right|^{3}}{2^{n m}}$.

3. Let us consider that instead of (1) we had to evaluate the number $J$ of $f_{1}, f_{2}, f_{3}$ such that $\forall i, 1 \leq i \leq m, S_{i}=f_{3}\left(Y_{i}\right)$ with $Y_{i}=R_{i} \oplus f_{2}\left(L_{i} \oplus f_{1}\left(R_{i}\right)\right)$ (i.e. here we do not have the term $L_{i} \oplus f_{1}\left(R_{i}\right)$ ). Then, for random $L_{i}, R_{i}$ and for random $f_{1}, f_{2}, f_{3}$, we will have about 2 times more collisions $S_{i}=S_{j}$ compared with a random variable $S_{i}$. So if $S_{i}$ is random, $J \ll \frac{\left|F_{n}\right|^{3}}{2^{n m}}$ in this case. For (1) we will prove (among other results) that, unlike here for $J$, when the $S_{i}$ are random, we always have $H \simeq \frac{\left|F_{n}\right|^{3}}{2^{n m}}$.

Analysis of (1). (In appendix B an example is given on what we do here) We will consider that all the $Y_{i}$ are given (as well as the $L_{i}, R_{i}, S_{i}$ ), and we want to study how $H$ can depend on the values $S_{i}$. If $H$ has almost always the same value for all the $S_{i}$, then (by summation on all the $Y_{i}$ ) we will get $H \simeq \frac{\left|F_{n}\right|^{3}}{2^{n m}}$, and for all $\left[L_{i}, R_{i}\right]$ the $S_{i}$ will look random, as wanted, when $f_{1}, f_{2}, f_{3}$ are randomly chosen in $F_{n}$ (this is an indirect way to evaluate $H$ ).

In (1), when we have a new value $Y_{i}$, whatever $S_{i}$ is, $f_{3}$ is exactly fixed on this point $Y_{i}$ by (1). However if $Y_{i}$ is not a new value, we have $Y_{i}=Y_{j} \Rightarrow$ $L_{i} \oplus f_{1}\left(R_{i}\right)=L_{j} \oplus f_{1}\left(R_{j}\right) \oplus S_{i} \oplus S_{j}$. For each equation $Y_{i}=Y_{j}$, we will introduce a value $\lambda_{k(i, j)}=S_{i} \oplus S_{j}$. We want to evaluate the number $H^{\prime}$ of $\left(f_{1}, f_{2}\right)$ such that: $\forall i, 1 \leq i \leq m, f_{2}\left(L_{i} \oplus f_{1}\left(R_{i}\right)\right)=R_{i} \oplus Y_{i}$

We will fix the points $(i, j)$ where $X_{i}=X_{j}$, i.e. we look for solutions $\left(f_{1}, f_{2}\right)$ such that $X_{i}=X_{j}$ exactly on these $(i, j)$, and, again, we want to evaluate how the number $H^{\prime}$ of $\left(f_{1}, f_{2}\right)$ can depend on the values $S_{i}$ (i.e. on the values $\lambda_{k}$ ).

We will group the equations $(2)$ by the same $f_{1}\left(R_{i}\right)$, i.e. by "blocks in $R, X, Y$ ": two indices $i$ and $j$ are in the same block if we can go from $i$ to $j$ by equations $R_{k}=R_{l}$, or $X_{k}=X_{l}$, or $Y_{k}=Y_{l}$ (Since $X_{k}=X_{l} \Rightarrow f_{1}\left(R_{k}\right)=$ $f_{1}\left(R_{l}\right) \oplus L_{k} \oplus L_{l}$ and $Y_{k}=Y_{l} \Rightarrow f_{1}\left(R_{k}\right)=f_{1}\left(R_{l}\right) \oplus L_{k} \oplus L_{l} \oplus \lambda_{k(i, j)}$, from these relations, we can replace the variable $f_{1}\left(R_{k}\right)$ by the variable $f_{1}\left(R_{l}\right)$ instead). 
Finally, the only dependencies on the $\lambda_{k}$ come when we want to evaluate the number $H^{\prime \prime}$ of $f_{1}$ such that: $\forall i, 1 \leq i \leq \alpha, X_{i}$ are pairwise distinct, where $\alpha$ is the number of $X_{i}$ that we want pairwise distinct (if wanted we can assume $\alpha \leq O\left(\frac{m^{2}}{2^{n}}\right)$ since variables with no equation in $R, X$ or $Y$ create no problem). Each $X_{i}$ has an expression like this: $X_{i}=f_{1}\left(R_{j}\right) \oplus \lambda_{k} \oplus L_{l}^{\prime}$ (where $L_{l}^{\prime}$ is an expression in $\oplus$ of some $L_{i}$ values), or like this: $X_{i}=f_{1}\left(R_{j}\right) \oplus L_{l}^{\prime}$. This gives a number of solutions for $f_{1}$ that depends only of the fact that some equations of degree one in the $\lambda_{k}$ variables are satisfied or not.

(These equations are $X_{i} \oplus X_{j}=X_{k} \oplus X_{l}$ where $i, j$ are in the same block in $R, X, Y$ and $k, l$ are in the same block in $R, X, Y$, so these equations can be written only the $L_{i}$ and $\lambda_{k}$ variables).

Example. In the example given in appendix B, $\lambda_{1}=L_{1} \oplus L_{4} \oplus L_{5} \oplus L_{7}$ is one of these equations, that can be true or not when the $\lambda_{i}$ values are fixed (here it comes from $\left.X_{1} \oplus X_{2} \oplus X_{5} \oplus X_{7}\right)$.

Analysis of the dependencies in the $\lambda_{k}$. First, we can notice that if the system has no solution due to an incompatibility (for example if we want $X_{1}=f_{1}\left(R_{1}\right) \oplus L_{1}$ and $X_{2}=f_{1}\left(R_{1}\right) \oplus \lambda_{1}$ to be distinct) then we have a circle in $R, X, Y$ with at least one equation in $Y$. The probability to get such circles has been evaluated in section 4 and is negligible if $m \ll 2^{n}$. So we will assume that we have no incompatibility in the system that says that the $X_{i}$ variables considered are pairwise distinct. Let $\mu$ be the number of variables $\lambda_{k}$ that satisfied at least one of these equations among the $\left(\frac{\alpha^{2}}{2}\right)$ equations considered for the evaluation of $f_{1}$. Each of the $\mu$ special $\lambda_{k}$ values can have at most $\alpha$ exceptional relations. So for a $\lambda$ like this, we have: $H \leq H^{*}\left(1-\frac{\alpha}{2^{n}}\right)^{-\mu}$. The value $\left(1-\frac{\alpha}{2^{n}}\right)^{-\mu}$ can be $\gg 1$, but since we have $\mu$ exceptional relations of degree one on $\mu$ variables $\lambda_{i}$, the weight $W_{\lambda}$ of these $\lambda$ values (i.e. the number of $f_{1}, f_{2}, f_{3}$ that give these values multiplied by the number of these values) satisfies:

$$
W_{\lambda} \leq \frac{1}{2^{n \mu}} C_{\frac{\alpha^{2}}{2}}^{\mu}\left(1-\frac{\alpha}{2^{n}}\right)^{-\mu} \text { (we denote by } A_{\mu} \text { this expression). }
$$

(since we have $\leq \frac{\alpha^{2}}{2}$ possible equations). We have:

$$
A_{\mu+1} \geq A_{\mu} \Leftrightarrow\left(\frac{\alpha^{2}}{2}-\mu\right)\left(1-\frac{\alpha}{2^{n}}\right)^{-1} \geq 2^{n}(\mu+1) \Leftrightarrow \mu \leq \text { about } \frac{\alpha^{2}}{2 \cdot 2^{n}}
$$

So the weight $W_{\lambda}$ becomes negligible as soon as $\mu \gg \frac{\alpha^{2}}{2 \cdot 2^{n}}$.

Remark. If these $\mu$ variables $\lambda_{i}$ generate almost all the possible relations with these variables, then the weight of these variables is even smaller since we just have to choose these $\mu$ variables among the $\alpha$ variables and then they are fixed (since almost all the equations are satisfied, many of these equations give equivalent values for the special $\lambda_{i}$ ). So we will have a $C_{\alpha}^{\mu}$ instead of $C_{\frac{\alpha^{2}}{2}}^{\mu}$. 
Finally we have obtain:

Theorem 5.1 Let $\mathcal{F}$ be the set of values that we fix: i.e. in $\mathcal{F}$ we have the values of the $Y_{i}$, and all the indices $(i, j)$ where we have all the equations $X_{i}=X_{j}$. Then if $S$ and $S^{\prime}$ are two sequences of values of $I_{n}^{m}$ such that:

1. $\forall i, j, Y_{i}=Y_{j} \Rightarrow S_{i} \neq S_{j}\left(\right.$ and $\left.S_{i}^{\prime} \neq S_{j}^{\prime}\right)$.

2. No circle in $R, X, Y$ can be created from the equalities $Y_{i}=Y_{j} \Rightarrow S_{i} \oplus S_{j}=$ $X_{i} \oplus X_{j}$ and $R_{k}=R_{l} \Rightarrow X_{k} \oplus X_{l}=L_{k} \oplus L_{l}$.

Then the number $H_{\mathcal{F}}$ of $f_{1}, f_{2}, f_{3}$ solutions satisfies:

$$
\left|H_{\mathcal{F}}(S)-H_{\mathcal{F}}\left(S^{\prime}\right)\right| \leq H_{\mathcal{F}}(S) \cdot(q+r)
$$

where $q=\frac{m^{2}}{2 \cdot 2^{n}}$ comes from the $\lambda_{i}$ with very few special equalities, and $r$ is a very small term related to the weight of the $\lambda_{i}$ with a lot of special equalities (as we have seen $r$ is negligible when $m \ll 2^{n}$ ).

We can do the same for $\left[S_{i}, T_{i}\right]$, as we did for $\left[Y_{i}, S_{i}\right]$. So, since by summation, we must obtain all the $\left(f_{1}, \ldots, f_{4}\right)$ with no circles, from theorem 5.1 we will get our results. Here the set $E^{\prime}$ depends on $E$, so this works for non-adaptive attacks. For adaptive attacks see [15] (then we have to eliminate some equations by conditions in $\left[S_{i}, T_{i}\right]$ independently of $\left[L_{i}, R_{i}\right]$, or to study the expectancy of the deviation of $H$ ).

Remark. Another possibility is to use the result of [5]: with 2 times more rounds, security in CPA-1 can be changed in security in CPCA-2. However we would get like this CPCA-2 for 10 rounds (exactly as in [14]) instead of 6 rounds.

\section{Comparing [14] and This Paper}

Technically the main differences between [14] and this paper are:

1. Here we introduce a condition: no more than $\frac{\lambda m(m-1)}{2^{n}}$ indices $(i, j), i<j$ such that $X_{i}=X_{j}$ (instead of no more than $\theta$ pairwise distinct indices such that $X_{i_{1}}=X_{i_{2}}=\ldots=X_{i_{\theta}}$ of [14]). this gives us security when $m \ll 2^{n}$ (instead of $m \ll 2^{n(1-\varepsilon)}$ or $m \ll \frac{2^{n}}{n}$ of [14]).

2. In [14], 3 rounds are needed for half the variables to look random, and then 4 more rounds for the $\left[S_{i}, T_{i}\right]$. Here we show that the $S_{i}$ will look random after 4 rounds even if the $Z_{i}$ are public (with a probability near 1 when $m \ll 2^{n}$ ). So for the $T_{i}$ we can use the same result with only one more round. Like this, we need less rounds in this paper compared with [14].

3. In this paper we study $\lambda_{k}$ that come for $\Psi^{4}$ from $Y_{i} \oplus Y_{j}=0$ (or similarly $Z_{i} \oplus Z_{j}=0$ for $\Psi^{5}$ ) while in [14] all possible $\lambda_{k}$ can be fixed. 


\section{Part II: Best Found Attacks}

\section{Generic Attacks on $\Psi^{5}$}

We will present here the two best generic attacks that we have found on $\Psi^{5}$ :

1. A CPA-1 attack on $\Psi^{5}$ with $m \simeq 2^{n}$ and $\lambda=O\left(2^{n}\right)$ computations (This is an improvement compared with $m \simeq 2^{3 n / 2}$ and $\lambda=O\left(2^{3 n / 2}\right)$ of [13]).

2. A KPA on $\Psi^{5}$ with $m \simeq 2^{3 n / 2}$ and $\lambda=O\left(2^{3 n / 2}\right)$ computations (This is an improvement compared with $m \simeq 2^{7 n / 4}$ and $\lambda=O\left(2^{7 n / 4}\right)$ of [13]).

1. CPA-1 attack on $\Psi^{5}$.

Let us assume that $R_{i}=$ constant, $\forall i, 1 \leq i \leq m, m \simeq 2^{n}$. We will simply count the number $N$ of $(i, j), i<j$ such that $S_{i}=S_{j}$ and $L_{i} \oplus T_{i}=L_{j} \oplus T_{j}$. This number $N$ will be about double for $\Psi^{5}$ compared with a truly random permutation.

Proof:

If $S_{i}=S_{j}$,

$L_{i} \oplus T_{i}=L_{j} \oplus T_{j} \Leftrightarrow L_{i} \oplus Z_{i}=L_{j} \oplus Z_{j} \Leftrightarrow f_{1}\left(R_{1}\right) \oplus f_{3}\left(Y_{i}\right)=f_{1}\left(R_{1}\right) \oplus f_{3}\left(Y_{j}\right)$ $\Leftrightarrow f_{3}\left(R_{1} \oplus f_{2}\left(L_{i} \oplus f_{1}\left(R_{1}\right)\right)\right)=f_{3}\left(R_{1} \oplus f_{2}\left(L_{j} \oplus f_{1}\left(R_{1}\right)\right)\right) \quad$ (\#).

This will occur if $f_{2}\left(L_{i} \oplus f_{1}\left(R_{1}\right)\right)=f_{2}\left(L_{j} \oplus f_{1}\left(R_{1}\right)\right)$, or if these values are distinct but have the same images by $f_{3}$, so the probability is about two times larger.

Remarks

(a) By storing the $S_{i} \| L_{i} \oplus T_{i}$ values and looking for collisions, the complexity is in $\lambda \simeq O\left(2^{n}\right)$.

(b) With a single value for $R_{i}$, we will get very few collisions. However this attack becomes significant if we have a few values $R_{i}$ and for all these values about $2^{n}$ values $L_{i}$.

2. KPA on $\Psi^{5}$.

The CPA attack can immediately be transformed in a KPA: for random $\left[L_{i}, R_{i}\right]$, we will simply count the number $N$ of $(i, j), i<j$ such that $R_{i}=R_{j}$, $S_{i}=S_{j}$, and $L_{i} \oplus T_{i}=L_{j} \oplus T_{j}$. We will get about $\frac{m(m-1)}{2^{3 n}}$ such collisions for $\Psi^{5}$, and about $\frac{m(m-1)}{2 \cdot 2^{3 n}}$ for a random permutation. This KPA is efficient when $m^{2}$ becomes not negligible compared with $2^{3 n}$, i.e. when $m \geq$ about $2^{3 n / 2}$.

Remark. These attacks are very similar with the attacks on 5-round Feistel schemes described by Knudsen (cf [2]) in the case where (unlike us) $f_{2}$ and $f_{3}$ are permutations (therefore, not random functions). Knudsen attacks are based on this theorem:

Theorem 7.1 (Knudsen, see [2]) Let $\left[L_{1}, R_{1}\right]$ and $\left[L_{2}, R_{2}\right]$ be two inputs of a 5-round Feistel scheme, and let $\left[S_{1}, T_{1}\right]$ and $\left[S_{2}, T_{2}\right]$ be the outputs. Let us assume that the round functions $f_{2}$ and $f_{3}$ are permutations (therefore they are not random functions of $F_{n}$ ). Then, if $R_{1}=R_{2}$ and $L_{1} \neq L_{2}$, it is impossible to have simultaneously $S_{1}=S_{2}$ and $L_{1} \oplus L_{2}=T_{1} \oplus T_{2}$. 
Proof. This comes immediately from (\#) above.

\section{Generic Attacks on $\Psi^{k}$ Generators, $k \geq 6$}

$\Psi^{k}$ has always an even signature. This gives an attack in $2^{2 n}$ if we want to distinguish $\Psi^{k}$ from random permutations (see [13]) and if we have all the possible cleartext/ciphertext. In this appendix, we will present the best attacks that we know when we want to distinguish $\Psi^{k}$ from random permutations with an even signature, or when we do not have exactly all the possible cleartext/ciphertext.

1. KPA with $k$ even.

Let $(i, j)$ be two indices, $i \neq j$, such that $R_{i}=R_{j}$ and $S_{i} \oplus S_{j}=L_{i} \oplus L_{j}$. From [10] or [11] p.146, we know the exact value of $H$ in this case, when $k$ is even. We have:

$$
H=H^{*}\left(1+\frac{1}{2^{\left(\frac{k}{2}-2\right) n}}-\frac{1}{2^{\left(\frac{k}{2}-1\right) n}}-\frac{2}{2^{\frac{k n}{2}}}+\frac{1}{2^{(k-1) n}}\right)
$$

where

$$
H^{*}=\frac{\left|F_{n}\right|^{k}}{2^{2 n m}} \cdot \frac{1}{1-\frac{1}{2^{2 n}}}
$$

i.e. $H^{*}$ is the average value of $H$ on two cleartext/ciphertext. So there is a small deviation, of about $\frac{1}{2^{\left(\frac{k}{2}-2\right) n}}$, from the average value.

So in a KPA, when the $\left[L_{i}, R_{i}\right]$ are chosen at random, and if the $f_{i}$ functions are chosen at random, we will get slightly more $(i, j), i<j$, with $R_{i}=R_{j}$ and $S_{i} \oplus S_{j}=L_{i} \oplus L_{j}$ from a $\Psi^{k}$ (with $k$ even) than from a truly random permutation. This can be detected if we have enough cleartext/ciphertext pairs from many $\Psi^{k}$ permutations. In first approximation, these relations will act like independent Bernoulli variables (in reality the equations are not truly independent, but this is expected to create only a modification of second order).

If we have $N$ possibilities for $(i, j), i<j$, and if $X$ is the number of $(i, j)$, $i<j / R_{i}=R_{j}$ and $S_{i} \oplus S_{j}=L_{i} \oplus L_{j}$, we expect to have:

$$
\begin{aligned}
& E(X) \simeq \frac{N}{2^{2 n}} \\
& V(X) \simeq \frac{N}{2^{2 n}} \\
& \sigma(X) \simeq \frac{\sqrt{N}}{2^{n}} .
\end{aligned}
$$

We want $\sigma(X) \leq \frac{N}{2^{\left(\frac{k}{2}-2\right) n}} \cdot \frac{1}{2^{2 n}}$ in order to distinguish $\Psi^{k}$ from a random permutation. So we want $\frac{\sqrt{N}}{2^{n}} \leq \frac{N}{2^{\frac{k}{2} n}}$ i.e. $N \geq 2^{(k-2) n}$.

However, if we have $\mu$ available permutations, with about $2^{2 n}$ cleartext/ciphertext for each of these permutations, then $N \simeq 2^{4 n} \mu$ (here we know these $\mu$ permutations almost on every possible cleartext. If not, $\mu$ will be larger and we will do more computations). $N \geq 2^{(k-2) n}$ gives $\mu \geq 2^{(k-6) n}$. This is an attack with $2^{(k-6) n}$ permutations and $2^{2 n} \mu \simeq 2^{(k-4) n}$ computations.

2. KPA with $k$ odd.

In [15], a KPA with $k$ odd is given (it has the same properties as the attack above for $k$ even). 


\section{Conclusion}

For a block cipher from $2 n$ bits $\rightarrow 2 n$ bits, we generally want to have no better attack than attacks with $\geq 2^{2 n}$ computations. If this block cipher is a Feistel scheme we then need to have $\geq 6$ rounds since (as shown in this paper) there is a generic attack on 5 rounds with $2^{n}$ computations in CPA- 1 and $2^{3 n / 2}$ computations in KPA.

In this paper we have also shown that however, in the model where the adversaries have unlimited computing power but have access to only $m$ cleartext/ciphertext pairs, the maximum possible security (i.e. $m \ll 2^{n}$ ) is obtained already for 5 rounds for CPA- 1 and CPA-2 attacks. This solves an open problem of [1] and [14]. Moreover 6-round Feistel schemes can resist all CPCA-1 and CPCA-2 attacks when $m \ll 2^{n}$ (For CPCA-1 or CPCA-2 the case $k=5$ rounds is still unclear: we only know that the security is between $m \ll 2^{n / 2}$ and $m \ll 2^{n}$ ). When $2^{2 n}$ is small (for example to generate 1000 pseudorandom permutations with an even signature of 30 bits $\rightarrow 30$ bits) then more than 6 rounds are needed. In this paper we have studied such attacks, and we have extended the "coefficients $H$ technique" to various cryptographic attacks.

We think that our proof strategy is very general and should be also efficient in the future to study different kinds of functions or permutation generators, such as, for example, Feistel schemes with a different group law than $\oplus$, or unbalanced Feistel schemes.

\section{References}

1. W. Aiello and R. Venkatesan. Foiling Birthday Attacks in Length-Doubling Transformations-Benes: A Non-Reversible Alternative to Feistel. EUROCRYPT '96 (Lecture Notes in Computer Science 1070), pp. 307-320, Springer-Verlag.

2. L. R. Knudsen. DEAL - A 128 bit Block Cipher. Technical Report \#151, University of Bergen, Departement of Informatics, Norway, February 1998.

3. M. Luby and C. Rackoff. How to construct pseudorandom permutations from pseudorandom functions. SIAM Journal on Computing, vol. 17, no 2, pp. 373386, April 1988.

4. U. Maurer. A simplified and generalized treatment of Luby-Rackoff pseudorandom permutation generators. EUROCRYPT '92, pp. 239-255, Springer-Verlag.

5. U. Maurer. Indistinguishability of Random Systems. EUROCRYPT '02 (Lecture Notes in Computer Science 2332), pp. 110-132, Springer-Verlag.

6. U. Maurer and K. Pietrzak. The security of Many-Round Luby-Rackoff PseudoRandom Permutations. EUROCRYPT '03, pp. -, Springer-Verlag.

7. V. Nachev. Random Feistel schemes for $m=3$, available from the author at: Valerie.nachef@math.u-cergy.fr.

8. M. Naor and O. Reingold. On the Construction of pseudo-random perlutations: Luby-Rackoff revisited. Journal of Cryptology, vol. 12, 1999, pp. 29-66. Extended abstract was published in Proc. 29th Ann. ACM Symp. on Theory of Computing, 1997, pp. 189-199.

9. J. Patarin. Pseudorandom Permutations based on the DES Scheme. Eurocode '90, LNCS 514, pp. 193-204, Springer-Verlag. 
10. J. Patarin. New results on pseudorandom permutation generators based on the DES scheme. Crypto '91,pp. 301-312, Springer-Verlag.

11. J. Patarin. Etude des générateurs de permutations basés sur le schéma du DES. Ph. D. Thesis, Inria, Domaine de Voluceau, Le Chesnay, France, 1991.

12. J. Patarin. About Feistel Schemes with 6 (or More) Rounds. Fast Software Encryption 1998, pp. 103-121.

13. J. Patarin. Generic Attacks on Feistel Schemes. Asiacrypt '01 (Lecture Notes in Computer Science 2248), pp. 222-238, Springer-Verlag.

14. J. Patarin. Luby-Rackoff: 7 Rounds are Enough for $2^{n(1-\epsilon)}$ Security. Crypto '03 (Lecture Notes in Computer Science 2729), pp.513-529, Springer-Verlag.

15. J. Patarin. Extended version of this paper, avaible from the author.

16. B. Schneier and J. Kelsey. Unbalanced Feistel Networks and Block Cipher Design. FSE '96 (Lecture Notes in Computer Science 1039), pp. 121-144, Springer-Verlag.

\section{Appendices}

\section{A Summary of the Known Results on Random Feistel Schemes}

KPA denotes known plaintext attacks. CPA-1 denotes non-adaptive chosen plaintext attacks. CPA-2 denotes adaptive chosen plaintext attacks. CPCA-1 denotes non-adaptive chosen plaintext and ciphertext attacks. CPCA-2 denotes adaptive chosen plaintext and chosen ciphertext attacks. Non-Homogeneous properties are defined in [12].

This figure 1 present the best known results against unbounded adversaries limited by $m$ oracle queries.

\begin{tabular}{|c|c|c|c|c|c|c|}
\hline & KPA & CPA-1 & CPA-2 & CPCA-1 & CPCA-2 & Non-Homogeneous \\
\hline$\Psi$ & 1 & 1 & 1 & 1 & 1 & 1 \\
$\Psi^{2}$ & $2^{n / 2}$ & 2 & 2 & 2 & 2 & 2 \\
$\Psi^{3}$ & $2^{n / 2}$ & $2^{n / 2}$ & $2^{n / 2}$ & $2^{n / 2}$ & 3 & 2 \\
$\Psi^{4}$ & $2^{n}$ & $2^{n / 2}$ & $2^{n / 2}$ & $2^{n / 2}$ & $2^{n / 2}$ & 2 \\
$\Psi^{5}$ & $2^{n}$ & $2^{n}$ & $2^{n}$ & $\geq 2^{n / 2}$ & $\geq 2^{n / 2}$ & 2 \\
$\Psi^{6}$ & $2^{n}$ & $2^{n}$ & $2^{n}$ & $2^{n}$ & $2^{n}$ & $4^{*}$ \\
$\Psi^{k}, k \geq 6$ & $2^{n}$ & $2^{n}$ & $2^{n}$ & $2^{n}$ & $2^{n}$ & $\leq\left(\frac{k}{2}-1\right)^{2} * *$ \\
\hline
\end{tabular}

Fig. 1. Minimum number $m$ of queries to distinguish $\Psi^{k}$ from a random permutation of $I_{n} \rightarrow I_{n}$. For simplicity we denote $2^{\alpha}$ for $O\left(2^{\alpha}\right)$ i.e. when we have security as long as $m \ll 2^{\alpha}$. $\geq$ means best security proved.

$* \leq 4$ comes from [13] and $\geq 4$ comes from [7].

** with $k$ even and with $(k-2)(k-4)$ exceptional equations, so if $k \geq 7$ we need more than one permutation for this property. 


\begin{tabular}{|c|c|c|c|c|c|}
\hline & KPA & CPA-1 & CPA-2 & CPCA-1 & CPCA-2 \\
\hline$\Psi$ & 1 & 1 & 1 & 1 & 1 \\
$\Psi^{2}$ & $2^{n / 2}$ & 2 & 2 & 2 & 2 \\
$\Psi^{3}$ & $2^{n / 2}$ & $2^{n / 2}$ & $2^{n / 2}$ & $2^{n / 2}$ & 3 \\
$\Psi^{4}$ & $2^{n}$ & $2^{n / 2}$ & $2^{n / 2}$ & $2^{n / 2}$ & $2^{n / 2}$ \\
$\Psi^{5}$ & $\leq 2^{3 n / 2}$ & $2^{n}$ & $2^{n}$ & $\leq 2^{n}$ & $\leq 2^{n}$ \\
$\Psi^{6}$ & $\leq 2^{2 n}$ & $\leq 2^{2 n}$ & $\leq 2^{2 n}$ & $\leq 2^{2 n}$ & $\leq 2^{2 n}$ \\
$\Psi^{7}$ & $\leq 2^{3 n}$ & $\leq 2^{3 n}$ & $\leq 2^{3 n}$ & $\leq 2^{3 n}$ & $\leq 2^{3 n}$ \\
$\Psi^{8}$ & $\leq 2^{4 n}$ & $\leq 2^{4 n}$ & $\leq 2^{4 n}$ & $\leq 2^{4 n}$ & $\leq 2^{4 n}$ \\
$\Psi^{k}, k \geq 6 *$ & $\leq 2^{(k-4) n}$ & $\leq 2^{(k-4) n}$ & $\leq 2^{(k-4) n}$ & $\leq 2^{(k-4) n}$ & $\leq 2^{(k-4) n}$ \\
\hline
\end{tabular}

Fig. 2. Minimum number $\lambda$ of computations needed to distinguish a generator $\Psi^{k}$ (with one or many such permutations available) from random permutations with an even signature of $I_{n} \rightarrow I_{n}$. For simplicity we denote $\alpha$ for $O(\alpha)$. $\leq$ means best known attack.

* If $k \geq 7$ these attacks analyze about $2^{(k-6) n}$ permutations of the generator and if $k \leq 6$ only one permutation is needed.

History for $\Psi^{5}$. For $\Psi^{5}$ the best results of security against CPA-2 was:

- In 1988: $m \ll 2^{n / 2}$ (cf [3]).

- In 1998: $m \ll 2^{3 n / 4}(\operatorname{cf}[12])$.

- In 2003: $m \ll 2^{5 n / 6}$ (cf [13]).

- In 2004: $m \ll 2^{n}$ (cf this paper).

However CPCA-2 for $\Psi^{5}$ is still unclear: so far we only have the original result of Luby and Rackoff $m \ll 2^{n / 2}$.

\section{B Example for Theorem 3.1}

We will illustrate here theorem 3.1 on a small toy example. Let $1,2,3,4,5,6,7$ be our indices $(m=7)$. Let us assume that $f_{1}$ is fixed such that $R_{4}=R_{1}$, and $R_{7}=R_{5}$, are our only equations $R_{i}=R_{j} i>j$. Let us assume that the $Y_{i}$ are given, and that $Y_{4}=Y_{2}$, and $Y_{7}=Y_{3}$ are the only equations $Y_{i}=Y_{j}$, $i>j$.Then we want to show that $\lambda_{1}$ and $\lambda_{2}$ look random, where $\lambda_{1}=X_{4} \oplus X_{2}$ and $\lambda_{2}=X_{7} \oplus X_{3}$ when $f_{1}, f_{2}$ are randomly chosen. For this, we fix $\lambda_{1}$ and $\lambda_{2}$, $\lambda_{1} \neq 0, \lambda_{2} \neq 0$, and we look for the number $H$ of $\left(f_{1}, f_{2}\right)$ that give these values. We want to prove that this number $H$ does not depend significantly on $\lambda_{1}$ and $\lambda_{2}$ (except for well detected values of small weight). $H$ is the number of $\left(f_{1}, f_{2}\right)$ such that (here we put only pairwise distinct $R_{i}$ variables):

1. $f_{1}\left(R_{2}\right)=f_{1}\left(R_{1}\right) \oplus L_{2} \oplus L_{4} \oplus \lambda_{1}$ and $f_{1}\left(R_{5}\right)=f_{1}\left(R_{3}\right) \oplus L_{3} \oplus L_{7} \oplus \lambda_{2}$ (these two equations do not create any problem: they just fix $f_{1}$ on two points).

2. Block $R_{1} Y$ :

$f_{2}\left(L_{1} \oplus f_{1}\left(R_{1}\right)\right)=R_{1} \oplus Y_{1}$

$f_{2}\left(L_{4} \oplus \lambda_{1} \oplus f_{1}\left(R_{1}\right)\right)=R_{2} \oplus Y_{2}$

$f_{2}\left(L_{4} \oplus f_{1}\left(R_{1}\right)\right)=R_{1} \oplus Y_{2}$. 


\section{Block $R_{3} Y$ :}

$f_{2}\left(L_{3} \oplus f_{1}\left(R_{3}\right)\right)=R_{3} \oplus Y_{3}$

$f_{2}\left(L_{3} \oplus L_{5} \oplus L_{7} \oplus \lambda_{2} \oplus f_{1}\left(R_{3}\right)\right)=R_{5} \oplus Y_{5}$

$f_{2}\left(L_{3} \oplus f_{1}\left(R_{3}\right) \oplus \lambda_{2}\right)=R_{5} \oplus Y_{3}$.

\section{$\underline{\text { Block } R_{6} Y \text { : }}$}

$$
f_{2}\left(L_{6} \oplus f_{1}\left(R_{6}\right)\right)=R_{6} \oplus Y_{6}
$$

Let us assume that, for example, all the $R_{i} \oplus Y_{i}$ are pairwise distinct. Then we want to evaluate the number of functions $f_{1}$ such that all the $X_{i}$ are pairwise distinct. These conditions are more difficult to analyze since here we do not want equalities, but non equalities.

- If $\lambda_{1} \in\left\{0, L_{1} \oplus L_{4}\right\}$, or $\lambda_{2} \in\left\{0, L_{5} \oplus L_{7}\right\}$, we have no solution (these values give a circle in $R, X, Y)$.

- For the $X_{i}$ to be pairwise distinct, we must choose $f_{1}$ such that: $f_{1}\left(R_{1}\right) \oplus$ $f_{1}\left(R_{3}\right)$ is not in $A$, where $A$ is a set of 9 values (or less if we have collisions): $A=\left\{L_{1} \oplus L_{3}, L_{4} \oplus \lambda_{1} \oplus L_{3}, L_{4} \oplus L_{3}, L_{1} \oplus L_{3} \oplus L_{5} \oplus L_{7} \oplus \lambda_{2}, L_{4} \oplus \lambda_{1} \oplus L_{3} \oplus L_{5} \oplus\right.$ $\left.L_{7} \oplus \lambda_{2}, L_{4} \oplus L_{3} \oplus L_{5} \oplus L_{7} \oplus \lambda_{2}, L_{1} \oplus L_{3} \oplus \lambda_{2}, L_{4} \oplus \lambda_{1} \oplus L_{3} \oplus \lambda_{2}, L_{4} \oplus L_{3} \oplus \lambda_{2}\right\}$. In the proof of theorem 3.1, we analyze the possible dependencies of $|A|$ with the $\lambda_{i}$ values.

\section{Examples of Unusual Values of $\boldsymbol{H}$ for $\Psi^{5}$}

Example 1: Large value for $H$

With $m=2$, when $R_{1}=R_{2}, S_{1}=S_{2}$ and $L_{1} \oplus L_{2}=T_{1} \oplus T_{2}$, then

$$
H=\frac{\left|F_{n}\right|^{5}}{2^{2 n m}}\left(2-\frac{1}{2^{n}}\right) \text {. }
$$

So here the value of $H$ is about double than average with only $m=2$.

Remark: $\forall k \in \mathbf{N}^{*}, \Psi^{k}$ has always such large $H$ with small $m\left(m \leq\left(\frac{k}{2}-1\right)^{2}\right.$ if $k$ is even), we say that " $\Psi^{k}$ is not homogeneous": see [12]. However, when $k \geq 7$, the probability that such inputs/outputs exist is generally negligible if we study only one single specific permutation.

Example 2: Small value for $H$

Here our example cannot be with $m \ll 2^{n / 2}$ since we know that we always have

$$
H \geq \frac{\left|F_{n}\right|^{5}}{2^{2 n m}}\left(1-\frac{m(m-1)}{2^{n}}\right)
$$

(the proof is the same for $\Psi^{4}$ and $\Psi^{5}$ ).

However, we will show that when $m \rightarrow 2^{n / 2}, H$ can be much smaller than average (i.e. $m \rightarrow 2^{n}$ is not necessary, $m \rightarrow 2^{n / 2}$ is enough). In this example 2 , we will assume: 
1. $\forall i, j, 1 \leq i \leq j \leq m, R_{i}=R_{j}\left(=R_{1}\right)$.

2. $\forall i, j, 1 \leq i \leq j \leq m, S_{i}=S_{j}\left(=S_{1}\right)$.

3. $\forall i, j, 1 \leq i \leq j \leq m, i \neq j \Rightarrow L_{i} \oplus L_{j} \neq T_{i} \oplus T_{j}$ (in example 3 below we will not need this condition 3 ).

To get condition 3, we may assume, for example, that $\forall i, 1 \leq i \leq m, L_{i}=$ $i \oplus \varphi(i)$ and $T_{i}=\varphi(i)$, where $\varphi$ is well chosen. So $L_{i} \oplus L_{j}=T_{i} \oplus T_{j} \Leftrightarrow i=j$.

From 1 we have: $\forall i, j, 1 \leq i \leq j \leq m, X_{i} \oplus X_{j}=L_{i} \oplus L_{j}$.

From 2 we have: $\forall i, j, 1 \leq i \leq j \leq m, Z_{i} \oplus Z_{j}=T_{i} \oplus T_{j}$.

$H$ is the number of $f_{1}, f_{2}, f_{3}, f_{4}, f_{5}$ such that: $\forall i, 1 \leq i \leq m$,

$$
\begin{gathered}
L_{i} \oplus f_{1}\left(R_{1}\right)=X_{i} \\
R_{1} \oplus f_{2}\left(L_{i} \oplus f_{1}\left(R_{1}\right)\right)=Y_{i} \\
X_{i} \oplus f_{3}\left(Y_{i}\right)=Z_{i} \\
Y_{i} \oplus f_{4}\left(T_{i} \oplus f_{5}\left(S_{1}\right)\right)=S_{1} \\
Z_{i} \oplus f_{5}\left(S_{1}\right)=T_{i}
\end{gathered}
$$

So $H$ is $\left|F_{n}\right|^{2}$ times the number of $f_{2}, f_{3}, f_{4}$ such that: $\forall i, 1 \leq i \leq m$,

$$
\left\{\begin{array}{l}
Y_{i}=R_{1} \oplus f_{2}\left(L_{i} \oplus f_{1}\left(R_{1}\right)\right)=S_{1} \oplus f_{4}\left(T_{i} \oplus f_{5}\left(S_{1}\right)\right) \\
f_{3}\left(Y_{i}\right)=L_{i} \oplus T_{i} \oplus f_{1}\left(R_{1}\right) \oplus f_{5}\left(S_{1}\right)
\end{array}\right.
$$

Since all the $L_{i} \oplus T_{i}$ are pairwise distinct, all the $Y_{i}$ must be pairwise distinct. So for $Y_{i}, 1 \leq i \leq m$, we have exactly: $2^{n}\left(2^{n}-1\right)\left(2^{n}-2\right) \ldots\left(2^{n}-m+1\right)$ solutions.

Now when $Y_{i}, 1 \leq i \leq m$, are fixed, $f_{2}, f_{3}$ and $f_{4}$ are fixed on exactly $m$ pairwise distinct points. So $H=\frac{\left|F_{n}\right|^{5}}{2^{3 n m}} 2^{n}\left(2^{n}-1\right)\left(2^{n}-2\right) \ldots\left(2^{n}-m+1\right)$.

Let $H^{*}$ be the average value of $H$ (when the $\left[S_{i}, T_{i}\right]$ are pairwise distinct).

$$
H^{*}=\frac{\left|F_{n}\right|^{5}}{2^{2 n}\left(2^{2 n}-1\right)\left(2^{n}-2\right) \ldots\left(2^{2 n}-m+1\right)} \geq \frac{\left|F_{n}\right|^{5}}{2^{2 n m}} .
$$

So here:

$$
\begin{gathered}
\frac{H}{H^{*}} \leq\left(1-\frac{1}{2^{n}}\right)\left(1-\frac{2}{2^{n}}\right) \ldots\left(1-\frac{m-1}{2^{n}}\right) \\
\ln \left(\frac{H}{H^{*}}\right) \simeq-\frac{1+2+\ldots+(m-1)}{2^{n}}=-\frac{m(m-1)}{2^{n}} .
\end{gathered}
$$

So when $m(m-1)$ is not negligible compared with $2^{n}, H$ will be significatively smaller than $H^{*}$, as claimed.

Remark 1. Here $R_{i} \oplus S_{i}$ is not random (since $R_{i} \oplus S_{i}$ is constant), and $L_{i} \oplus T_{i}$ is not random (in example 3 below we will remove this condition on $L_{i} \oplus T_{i}$ ). These hypothesis are generally unrealistic in a cryptographic attack, where $\forall i$, $1 \leq i \leq m, L_{i}$ or $T_{i}$, and $R_{i}$ or $S_{i}$, cannot be chosen. 
Remark 2. If we start, as here, from $\left[L_{i}, R_{i}\right]$ values with $R_{i}$ constant, then the $X_{i}$ values are pairwise distinct, so the $Y_{i}$ values are perfectly random (if we define $Y_{i}$ only from the relation $\left.Y_{i}=R_{i} \oplus f_{2}\left(X_{i}\right)\right)$. However, the $Z_{i}$ values are not perfectly random (since the probability to have $Z_{i} \oplus Z_{j}=L_{i} \oplus L_{j}$ is the probability to have $f_{3}\left(Y_{i}\right)=f_{3}\left(Y_{j}\right)$ so is about double than average). Similarly, the $\left[S_{i}, T_{i}\right]$ values are not perfectly random since the probability to have $S_{i}=S_{j}$ and $T_{i} \oplus T_{j}=L_{i} \oplus L_{j}$ is in relation with the probability to have $f_{3}\left(Y_{i}\right)=f_{3}\left(Y_{j}\right)$, so is about double than average. We will use again this idea in example 3 below.

Remark 3. Here when $m \rightarrow 2^{n / 2}$, we can have circles in $Y, S$, (and circles in $R, Y$ ) and this is a way to explain why in this example $H$ can be much smaller than $H^{*}$.

Example 3: Small value for $H$, with random $L_{i}$ and $T_{i}$

In this example 3, we will assume:

1. $\forall i, j, 1 \leq i \leq j \leq m, R_{i}=R_{j}\left(=R_{1}\right)$.

2. $\forall i, j, 1 \leq i \leq j \leq m, S_{i}=S_{j}\left(=S_{1}\right)$.

3. Let $A_{i}=L_{i} \oplus T_{i}$. Then $A_{i}, 1 \leq i \leq m$, is random. More precisely it will be enough to assume that the number $N$ of collisions $A_{i}=A_{j}, i<j$, is $<\frac{m(m-1)}{2 \cdot 2^{n} \ln 2}$ to show that $H$ is small compared with the average value $H^{*}$. For random values $A_{i}$ we have $N \simeq \frac{m(m-1)}{2 \cdot 2^{n}}$, so it is the case $\left(\frac{1}{\ln 2} \simeq 1,44\right)$.

As in example 2, $H$ is $\left|F_{n}\right|^{2}$ times the number of $f_{2}, f_{3}, f_{4}$ such that: $\forall i$, $1 \leq i \leq m$

$$
\left\{\begin{array}{l}
Y_{i}=R_{1} \oplus f_{2}\left(L_{i} \oplus f_{1}\left(R_{1}\right)\right)=S_{1} \oplus f_{4}\left(T_{i} \oplus f_{5}\left(S_{1}\right)\right) \\
f_{3}\left(Y_{i}\right)=L_{i} \oplus T_{i} \oplus f_{1}\left(R_{1}\right) \oplus f_{5}\left(S_{1}\right)
\end{array} .\right.
$$

Since all the $L_{i} \oplus f_{1}\left(R_{1}\right)$ are pairwise distinct, and all the $T_{i} \oplus f_{5}\left(S_{1}\right)$ are pairwise distinct, $f_{2}$ and $f_{4}$ are fixed on exactly $m$ points when $Y_{i}, 1 \leq i \leq m$, is fixed.

So $H$ is $\frac{\left|F_{n}\right|^{4}}{2^{2 n m}}$ times the number of $Y_{i}, f_{3}$ such that: $\forall i, 1 \leq i \leq m, f_{3}\left(Y_{i}\right)=$ $L_{i} \oplus T_{i} \oplus f_{1}\left(R_{1}\right) \oplus f_{5}\left(S_{1}\right)$.

Let $A_{i}$ be a sequence of values of $I_{n}, 1 \leq i \leq m$. We want to evaluate the number $h$ of $Y_{i}, f_{3}$ such that: $\forall i, 1 \leq i \leq m, f_{3}\left(Y_{i}\right)=A_{i}$. Let $h^{*}$ be the average value for $h$ (average on all sequences $A_{i}$ ). We have $h^{*}=\left|F_{n}\right|$. For random values $Y_{i}$, and random functions $f_{3}, A_{i}$ will have about 2 times more collisions $A_{i}=A_{j}$, $i<j$, than average sequences $A_{i}$.

So $h$ for random values $A_{i}$ is $\ll h^{*}$, and $h$ for values $A_{i}$ with 2 times more collisions than average is $\gg h^{*}$. This shows that if in this example $3 L_{i} \oplus T_{i}$ is random, then $H \ll H^{*}$. 